\title{
Giant area of transient hepatic attenuation difference, mimicking incidentaloma at liver ultrasound
}

\author{
Gaspare Parrinello • Daniele Torres • \\ Giuseppe Licata
}

Received: 23 April 2010/Accepted: 12 May 2010/Published online: 30 June 2010

(C) SIMI 2010

Concurrent with recent advances in diagnostic imaging techniques, the incidental finding of liver tumors, or incidentalomas, is increasing in asymptomatic and healthy individuals [1]. A 56-year-old healthy man underwent an abdomen ultrasound for a clinical check-up. A diffuse bright liver echo-pattern (indicating a low-moderate grade steatosis) and an unusual image (diameter approximately $10 \mathrm{~cm}$ ) in the VI-VII hepatic segment, characterized by a massive giant hypoechoic lesion with poorly defined margins and a near rounded hyperechoic area (diameter about $4 \mathrm{~cm}$ ) in the context were found (Fig. 1). The ultrasonographer suspected an angioma as first diagnostic hypothesis; however the large hypoechoic area around it, was not unique interpretation. In order to clarify the nature of the lesion, a contrast-enhancement examination was indicated to exclude an incidentaloma. Contrast-enhanced ultrasound revealed an irregularly circular lesion, characterized by early arterial nodular enhancement with delayed centripetal fill-in during the arterial phase. At 36 seconds, the lesion had almost completely filled in. At 45 seconds, the lesion was completely enhanced and sustained enhancement was observed in the late phase scan. The picture was suggestive for hepatic angioma (Fig. 2). Contrast-enhanced MultiSlice computed tomography (CT scan) (Fig. 3) was performed to confirm the diagnosis that proved to be an angioma in the VI hepatic segment (diameter about 4.6 $\mathrm{cm}$ ), revealing in contour a segmental area of transiently

G. Parrinello $\cdot$ D. Torres $(\bowtie) \cdot$ G. Licata

Biomedical Department of Internal and Specialty Medicine, Di.Bi.Mi.S., Internistic and Cardiovascular Diagnostic Ultrasound Laboratory, A.O.U Policlinico Paolo Giaccone, University of Palermo, Piazza delle Cliniche 2, 90127 Palermo, Italy

e-mail: daniele_torres@libero.it increased enhancement during the arterial phase, with isodense aspect to hepatic parenchyma on venous phase thus suggesting transient hepatic attenuation differences (THAD).

THAD is a hepatic perfusion anomaly, caused by an aberrant dual blood supply associated with numerous liver disorders, and it is visible on a contrast-enhanced biphasic spiral CT scan as parenchymal areas of high attenuation in the arterial phase, becoming an isodense image with the normal parenchyma in the portal venous phase [2]. Occasionally, attenuation differences may persist up to the portal venous phases due to a concurrent obstruction of a hepatic vein branch. THADs can be confused with tumor lesions, and are a compensatory relationship between two liver sources of blood supply so that the arterial flow increases as the portal flow decreases. In other words, this particular condition, also called steal syndrome, is seen when there are extensive anastomoses between two vascular beds, and the arterial supply to one is stenosed or occluded, resulting in diversion of blood to the other vascular bed. This is a result of communication between the main vessels, sinusoids, and peribiliary venules, which open in response to the autonomic nervous system, and humoral factors activated by the liver's demand for oxygen and metabolites. These lesions do not cause a mass effect on the vascular structures, and normal hepatic vascular structures can be seen passing through them. The lesions can be encountered in an entire lobe (lobar), segment (segmental), subsegment (subsegmental) or in a sub-capsular area of the liver. THADs may be noted to accompany hypervascular tumors, generally malignant but also benign (hepatocellular carcinoma, hypervascular metastases, cholangiocarcinoma, hemangioma, focal nodular hyperplasia, pyogenic abscess and focal eosinophilic necrosis) due to a steal syndrome [3]. However, they may also occur in patients without hepatic focal 


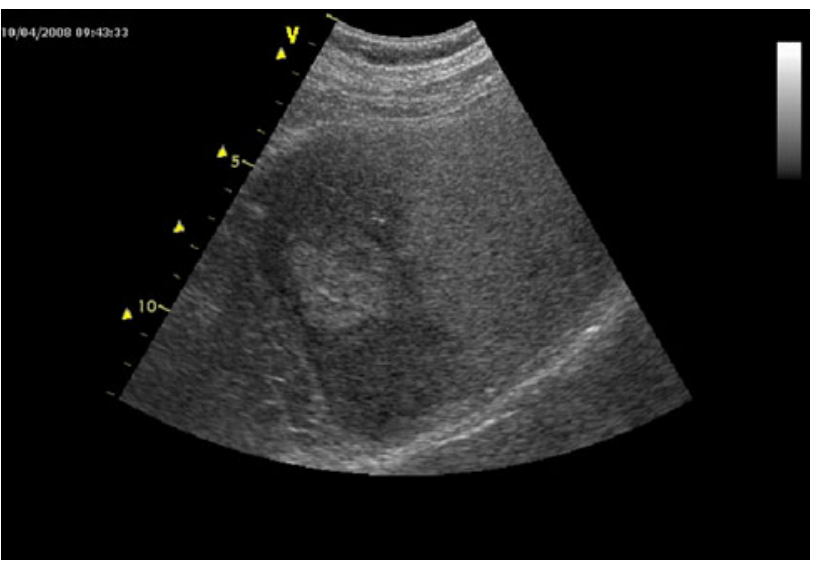

Fig. 1 Basal liver ultrasound

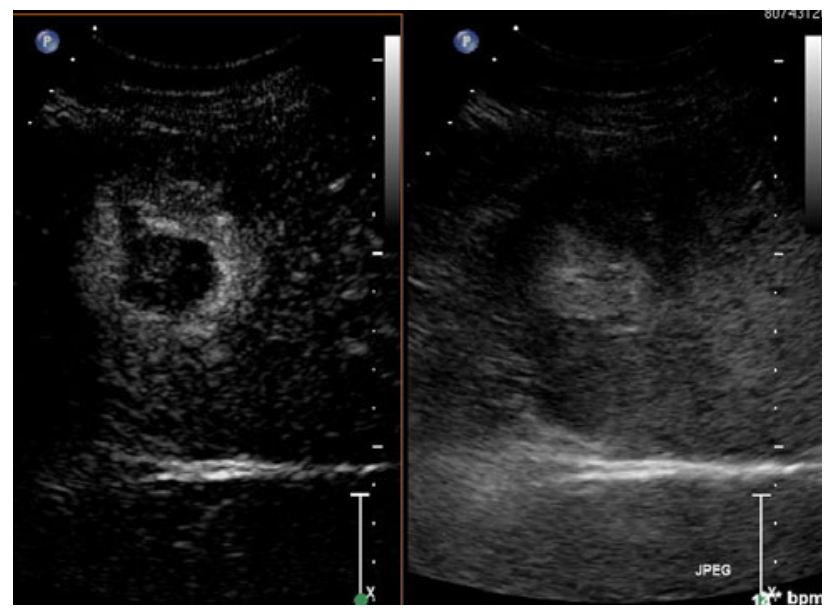

Fig. 2 Contrast-enhanced ultrasound

lesions. In accord with the current literature, it is possible to classify THADs, which are unrelated to focal hepatic lesions, as follows. From the pathophysiological point of view, we recognize four causes: portal vein stoppageobstruction, portal in-flow diversion, trauma and inflammation. When THADs are related to the first three causes, pathogenesis is portal hypoperfusion. Regarding the fourth group, the mediators of the arterial phenomena are those of inflammation even though portal hypoperfusion might be involved as well [4]. Causation for THADs not associated with focal lesions includes: cirrhosis (due to fibrosis and portal hypertension), Budd-Chiari syndrome, biliary obstruction or the dilatation of the entire biliary tree, trauma, arterioportal shunting, vascular compressions or invasion by tumors affecting the hepatic artery, the portal vein or hepatic vein (including obstruction due to vein thrombosis), iliac vein anomaly, occlusion of the superior vena cava (due to the diversion of flow into the paraumbilical veins), inflammatory changes (such as abscess,

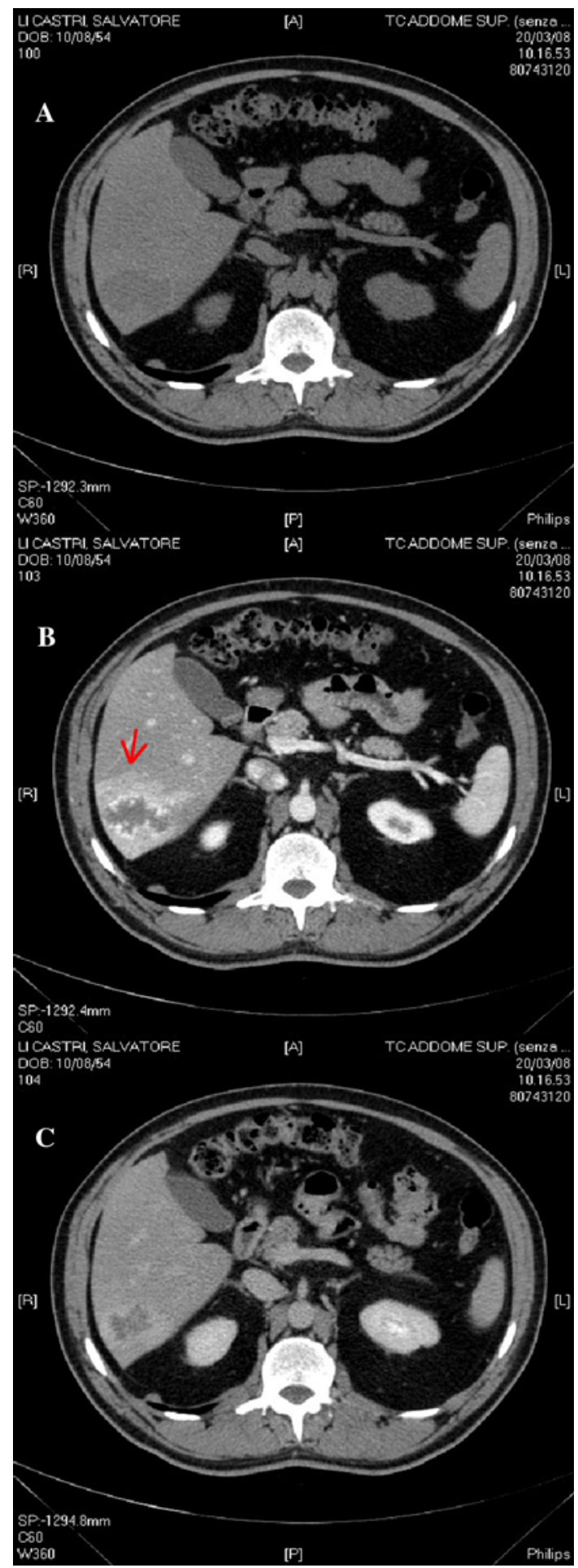

Fig. 3 Contrast-enhanced Multi-Slice CT: a basal phase, b arterial phase, $\mathbf{c}$ venous phase. The arrow in $\mathbf{b}$ indicates angioma and THAD in contour during the arterial phase of CT

cholangitis, chronic cholecystitis and acute inflammation of an adjacent organ), vascular variations (such as vascular vein, accessory cystic vein and aberrant gastric vein that may drain the systemic venous circulation into the liver), 
hepatic parenchymal compressions, previous biliary surgery, hepatic stasis caused by heart failure and constrictive pericarditis, fine-needle percutaneous biopsy, parenchymal compression by fractured ribs, a strengthened phrenic pillar, and transcatheter arterial chemoembolization (TACE) [58]. These lesions, typical of adolescent and adults, have also been recently described in neonates, frequently in infants $<1$ month of age than in the older age groups [9]. Nowadays, hepatic arterial phase evaluation may also be easily performed by magnetic resonance imaging (MRI), with which perfusion alterations can also be observed. We call these transient hepatic signal intensity differences (THID). An MRI may confirm any doubt regarding the diagnosis of THADs on a dynamic CT scan.

To conclude, THADs are benign entities associated to focal lesions (benign or malignant) or other diseases of the liver, characterized by hepatic hemodynamic changes that can be confused with tumoral lesions. They may be incidentally encountered on a dynamic CT scan as areas of increased parenchymal enhancement visible during the hepatic arterial phase only. However, they may also be seen during a liver ultrasound study, as in this case, and they can also accompany a typical hepatic angioma. The uniqueness of this finding is the presence of an uncommon, incidental, giant, hypoechoic focal hepatic lesion with neoplastic features, detectable on basal ultrasonography, and thereafter characterized with contrast enhancement, resulting in a THAD circuiting angioma by CT scan. Both clinicians and radiologists should be familiar with the US and CT scan appearances of this lesion in order to determine the etiology and pathogenesis of this lesion. THADs are important signs of a large, underlying variety of liver disorders, including malignancies. For this reason, a hypervascular cancerous focal lesion must always be excluded when performing the hepatic arterial phase. If THADs are still suspicious in the arterial phase, further examinations (MRI) should be performed to rule out clinically significant lesions.

Conflict of interest None.

\section{References}

1. Liu CL, Fan ST, Lo CM, Chan SC, Tso WK, Ng IO, Wong J (2004) Hepatic resection for incidentaloma. J Gastrointest Surg 8:785-793

2. Colagrande S, Centi N, Galdiero R, Ragozzino A (2007) Transient hepatic intensity differences: part 1 , those associated with focal lesions. Am J Roentgenol 188:154-159

3. Köseoğlu K, Taşkin F, Ozsunar Y, Cildağ B, Karaman C (2005) Transient hepatic attenuation differences at biphasic spiral CT examinations. Diagn Interv Radiol 11:96-101

4. Colagrande S, Centi N, Carmignani L, Salvatore Politi L, Villari N (2003) Meaning and etiopathogenesis of sectorial transient hepatic attenuation differences (THAD). Radiol Med 105:540-547

5. Colagrande S, Centi N, Galdiero R, Ragozzino A (2007) Transient hepatic intensity differences: part 2, those not associated with focal lesions. Am J Roentgenol 188:160-166

6. Saxena AK, Aiyappan SK, Kalra N, Khadwal AR, Sodhi KS (2009) Anomaly of iliac veins: a rare cause of transient hepatic attenuation difference in a child. Pediatr Radiol 39:1242-1245

7. Pradella S, Centi N, La Villa G, Mazza E, Colagrande S (2009) Transient hepatic attenuation difference (THAD) in biliary duct disease. Abdom Imaging 34:626-633

8. Hwang SH, Yu JS, Chung J, Chung JJ, Kim JH, Kim KW (2008) Transient hepatic attenuation difference (THAD) following transcatheter arterial chemoembolization for hepatic malignancy: changes on serial CT examinations. Eur Radiol 18:1596-1603

9. Towbin AJ, Ying J, Fleck R (2009) Transient hepatic attenuation differences in neonates. Pediatr Radiol 39:798-803 